[19] N. Yanai, M. Yamamoto, and Akira Mohri, "Anti-sway control for wiresuspended mechanism based on dynamics compensation," in Proc. Int. Conf. Robot. Autom., 2002, pp. 4287-4292.

[20] W. Wenli, D. Baoyan, L. Hong, N. Rendong, and P. Bo, "Inverse kinematics model of parallel macro-micro manipulator system," Chin. Sci. Bulletin, vol. 45, no. 24, pp. 2221-2226, Dec. 2000.

[21] M. A. Rahimi, H. Hemam, and Y. F. Zheng, "Experimental study of a cable-driven suspended platform," in Proc. Int. Conf. Robot. Autom., 1999, pp. 2342-2347.

[22] J. W. Jenog, S. H. Kim, Y. K. Kwak, and C. C. Smith, "Development of a parallel wire mechanism for measuring position and orientation of a robot end-effector," Mechatronics, vol. 8, pp. 845-861, 1998.

[23] M. S. Ohwovoriole, "An extension of screw theory and its application to the automatic of industrial assemblies," Ph.D. dissertation, Dept. Mech. Eng., Stanford Univ., Palo Alto, CA, 1980.

[24] V. D. Nguyen, "Constructing force-closure grasp," in Proc. Int. Conf. Robot. Autom., 1986, pp. 1386-1373.

[25] V. D. Nguyen, "Constructing force-closure grasp in 3D," in Proc. Int Conf. Robot. Autom., 1987, pp. 240-245.

[26] A. J. Goldman and A. W. Tucker, "Polyhedral convex cones," in Linear Inequalities and Related Systems. Princeton, NJ: Princeton Univ. Press, 1956.

[27] S. Arimoto, Control Theory of Non-Linear Mechanical Systems: A Passivity-Based and Circuit-Theoretic Approach. London, U.K: Oxford Univ Press, 1996.

\section{Potential Fields for Maintaining Connectivity of Mobile Networks}

\author{
Michael M. Zavlanos, and George J. Pappas
}

\begin{abstract}
The control of mobile networks of multiple agents raises fundamental and novel problems in controlling the structure of the resulting dynamic graphs. In this paper, we consider the problem of controlling a network of agents so that the resulting motion always preserves the connectivity property of the network. In particular, the connectivity condition is translated to differentiable constraints on individual agent motion by considering the dynamics of the Laplacian matrix and its spectral properties. Artificial potential fields are then used to drive the agents to configurations away from the undesired space of disconnected networks while avoiding collisions with each other. We conclude by illustrating a class of interesting problems that can be achieved while preserving connectivity constraints.
\end{abstract}

Index Terms-Dynamic graphs, graph connectivity, Laplacian matrix, potential fields.

\section{INTRODUCTION}

Controlling dynamic graphs has recently emerged as a fundamental problem in the area of multiagent and multirobot systems. Motivations come from the area of controlling formations of ground or aerial vehicles with applications in air traffic control, satellite clustering, automatic highways, mobile robotics, and mobile sensor networks. One of the main goals in this area is to achieve a coordinated objective

Manuscript received May 4, 2006; revised November 6, 2006. This paper was recommended for publication by Associate Editor G. Sukhatme and Editor K. Lynch upon evaluation of the reviewers' comments. This work was supported in part by ARO MURI SWARMS Grant W911NF-05-1-0219 and the NSF ITR Grant 0324977.

The authors are with the GRASP Laboratory, Department of Electrical and Systems Engineering, University of Pennsylvania, Philadelphia, PA 19104 USA (e-mail: zavlanos@grasp.upenn.edu; pappasg@grasp.upenn.edu).

Digital Object Identifier 10.1109/TRO.2007.900642 while using only relative information concerning positions and velocities. In this paper, the objective is to investigate the maintainenance of connectivity of the underlying graph.

Due to their frequent appearance in the area of multiagent systems, dynamic graphs have already received considerable attention. In [2], a measure of local connectedness of a network, which under certain conditions is sufficient for global connectedness, is introduced. This approach is distributed in the sense that this measure depends on neighbor-to-neighbor communication only. Motivated by a class of problems associated with control of distributed dynamic systems is also [3], where the authors consider a controllability framework for state-dependent dynamic graphs. In [4], the problem of finding the graph that corresponds to the maximum second smallest eigenvalue of its Laplacian is investigated. The authors propose a method that searches the graph space towards the direction that maximizes the second smallest eigenvalue of the graph Laplacian, and prove local convergence of their method. The second smallest eigenvalue of the graph Laplacian has also emerged as an important parameter in many system and control problems defined over networks [5]-[7]. In fact, it has been observed recently that this eigenvalue is a measure of stability and robustness of the networked dynamic system [6], [7].

Other research areas closely related to the problems discussed in this paper are formation stabilization [5]-[17], and in particular, consensus seeking by autonomous agents [5]-[7], as well as coverage tasks [18]. One of the main goals in formation stabilization and consensus problems is convergence of the agents to a common velocity. Various approaches have been studied, such as, control laws that involve graph Laplacians for the fixed (or switched) associated neighborhood graphs and artificial potential functions [7] or Lyapunov function methods [9], where the notion of "formation feedback" as a means to regulate agent motion in order to satisfy the global formation constraints, was also introduced. Navigation functions for distributed formation stabilization with collision avoidance constraints are used in [10] and [11], whereas necessary and sufficient conditions for achieving consensus are investigated in [6].

Motivated by the importance of connectivity in mobile sensor networks, as well as the connectivity assumption often made in formation stabilization or consensus problems, in this paper, we consider graph connectivity as our primary objective. As in our previous work [1], we consider the problem of designing controllers for multiagent systems that maintain connectivity of their underlying graphs. In contrast to the open loop controllers based on the solution of optimization problems [1], [4], we propose a centralized feedback control framework based on artificial potential fields. In the absence of agent failures and under the assumption that the initial graph is connected, graph connectivity is guaranteed for all time. The idea is to model connectivity as an imaginary obstacle in the free space, and use artificial potential fields to avoid collisions with it. Instead of the adjacency matrix and its dynamics [1], we use the Laplacian matrix of a graph and its spectral properties as a model for connectivity. Due to its closed-loop nature, our potential fields approach to maintain graph connectivity is more robust and easily extendible to accounting for secondary objectives, such as collision avoidance between the agents or the presence of formation leaders in the group.

The rest of this paper is organized as follows. In Section II, we define the network connectivity problem, and develop the necessary graphtheoretic background. In Section III, we deal with the technical issues of our approach, and propose the potential fields-based solution to the problem of maintaining connectivity of mobile networks. Finally, in Section IV, we discuss secondary objectives, such as collision avoidance and tracking leaders, and verify various connectivity tasks that illustrate the setting we have developed through computer simulations. 


\section{Problem Formulation}

\section{A. Graph-Theoretic Formulation}

Consider $n$ nodes in an $m$-dimensional space $\mathbb{R}^{m}$. We denote by $x_{i}(t) \in \mathbb{R}^{m}$ the coordinates of the $i$ th node at time $t$, where by convention, $x_{i}$ is considered an $m \times 1$ column vector, and by $\mathbf{x}(t)=\left[x_{1}^{T}(t) \ldots x_{n}^{T}(t)\right]^{T}$, the $m n \times 1$ vector resulting from stacking the coordinates of the nodes into a single vector. Suppose that the dynamics of the $i$ th node, for all $i \in\{1,2, \ldots, n\}$, are given by $\dot{x}_{i}(t)=u_{i}(t)$, where $u_{i}(t)$ is the control vector taking values in some set $U \subseteq \mathbb{R}^{m}$. In vector notation, the system dynamics become

$$
\dot{\mathbf{x}}(t)=\mathbf{u}(t)
$$

where $\dot{\mathbf{x}}(t)=\left[\dot{x}_{1}^{T}(t) \ldots \dot{x}_{n}^{T}(t)\right]^{T}$ and $\mathbf{u}(t)=\left[u_{1}^{T}(t) \ldots u_{n}^{T}(t)\right]^{T}$ are $m n \times 1$ vectors, respectively. The network of agents described by system (1), gives rise to a dynamic graph $\mathcal{G}(\mathbf{x}(t)) .{ }^{1}$

Definition 2.1: We call $\mathcal{G}(\mathbf{x}(t))=(\mathcal{V}, \mathcal{E}(\mathbf{x}(t)))$ a dynamic graph consisting of

- a set of vertices $\mathcal{V}=\{1, \ldots, n\}$ indexed by the set of mobile agents; and

- a set of edges $\mathcal{E}(\mathbf{x}(t))=\left\{(i, j) \mid d_{i j}(\mathbf{x}(t))<\delta\right\}$, with $d_{i j}(\mathbf{x}(t))=\left\|x_{i}(t)-x_{j}(t)\right\|_{2}$ as the Euclidean distance between agents $i$ and $j$, and $\delta>0$ as a constant.

Since we have control over the node (or vertex) dynamics, the question that naturally arises is whether we can control the motion of the agents, so that $\mathcal{G}(\mathbf{x}(t))$ satisfies a graph-theoretic property of interest for all time $t \geq 0$. In particular, we are interested in the connectivity property of a graph.

Definition 2.2 (Graph Connectivity): We say that a graph $\mathcal{G}$ is connected if there exists a path, i.e., a sequence of distinct vertices such that consecutive vertices are adjacent, between any two vertices in $\mathcal{G}$.

We denote by $\mathcal{C}_{n}$ the set of all connected graphs on $n$ vertices, and we address the problem of whether we can control the motion of all agents so that $\mathcal{G}(\mathbf{x}(t))$ always lies in the desired set $\mathcal{C}_{n}$ of connected graphs. More formally,

Problem 1 (Graph-Theoretic Formulation): Let $\mathcal{C}_{n}$ be the set of connected graphs, and assume any input to the graph $\mathcal{G}(\mathbf{x}(t))$ in the form of node motion. Given $\mathcal{C}_{n}$, determine a control law $\mathbf{u}(t)$ so that if $\mathcal{G}(\mathbf{x}(0)) \in \mathcal{C}_{n}$, then $\mathcal{G}(\mathbf{x}(t)) \in \mathcal{C}_{n}$ for all $t \geq 0$.

In other words, we would like the set $\mathcal{C}_{n}$ to be an invariant of motion for system (1). We achieve this goal by choosing an equivalent formulation using the algebraic representation of the dynamic graph $\mathcal{G}(\mathbf{x}(t))$.

\section{B. Algebraic Formulation}

The edge set of any dynamic graph can be associated with a set of weights such that for all $(i, j) \in \mathcal{E}(\mathbf{x}(t))$ the corresponding weight equals 1 and for all $(i, j) \notin \mathcal{E}(\mathbf{x}(t))$ the corresponding weight equals 0 . In matrix form, these weights consist the weighted adjacency matrix of a graph. The $0-1$ nature of these weights can be relaxed to allow for any positive weight for all $(i, j) \in \mathcal{E}(\mathbf{x}(t))$. This relaxation enables us to define smooth weight functions in our potential fields model. Hence, we have the following definition.

Definition 2.3 (Weighted Adjacency Matrix): Given a graph $\mathcal{G}$ with vertices $\mathcal{V}=\left\{v_{1}, \ldots, v_{n}\right\}$ and edges in the set $\mathcal{E}$, we define the weighted adjacency matrix of $\mathcal{G}$ to be the matrix $A=\left(a_{i j}\right)$ such that $a_{i j}>0$ if $\left(v_{i}, v_{j}\right) \in \mathcal{E}$, and $a_{i j}=0$ otherwise. Since we do not allow self-loops, for every $i \in\{1,2, \ldots, n\}$ we define $a_{i i}=0$.

\footnotetext{
${ }^{1}$ In the literature also referred to as a proximity graph due to the definition of the edge set.
}

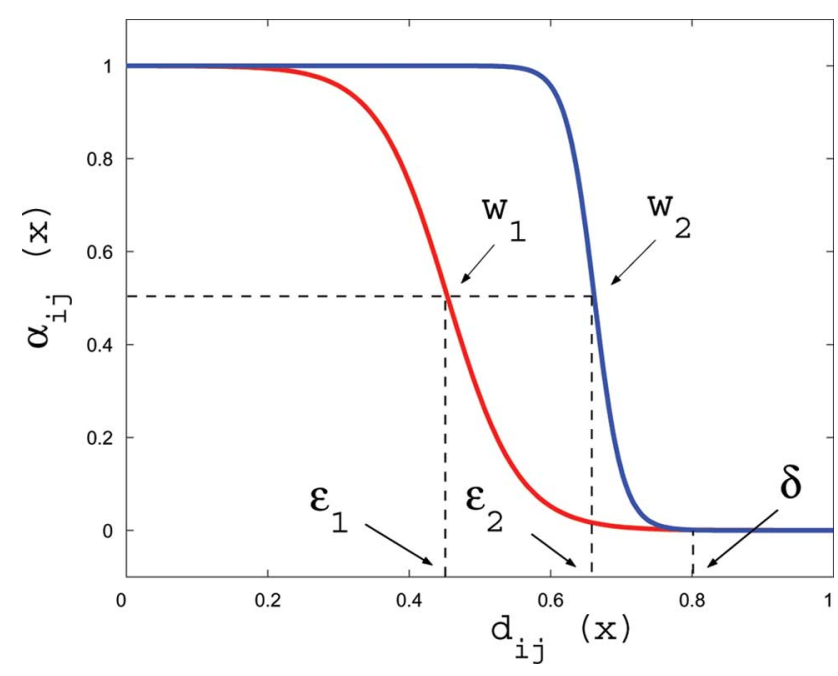

Fig 1. Weight function $a_{i j}(\mathbf{x})=\sigma_{w}\left(\epsilon-d_{i j}(\mathbf{x})\right)$ for $\delta=0.8$ and parameter values $w_{1}=20, \epsilon_{1}=0.4547$ and $w_{2}=50, \epsilon_{2}=0.6619$.

Note that if $A$ is an adjacency matrix of an undirected graph, as is the case we will be dealing with, then $A=A^{T}$. In order to capture the dependence of the edge set $\mathcal{E}(\mathbf{x}(t))$ on the state $\mathbf{x}(t)$, we need to introduce a dynamic adjacency matrix

$$
A(\mathbf{x}(t))=\left(a_{i j}(\mathbf{x}(t))\right)
$$

where $a_{i j}(\mathbf{x}(t))=\sigma_{w}\left(\epsilon-d_{i j}(\mathbf{x}(t))\right)$, and $\sigma_{w}(y)=1 /\left(1+e^{-w y}\right)$ is the sigmoid function with $w>0$ a constant (Fig. 1). ${ }^{2}$ Clearly, for any $\delta>0$ in Definition 2.1, there exist constants $w, \epsilon>0$ such that $a_{i j}(\mathbf{x}(t))>0$ for $d_{i j}(\mathbf{x}(t))<\delta$ and $a_{i j}(\mathbf{x}(t)) \rightarrow 0$ for $d_{i j}(\mathbf{x}(t)) \geq$ $\delta$. Hence, (2) is, pointwise in $\mathbf{x}(t)$, consistent with Definition 2.3 of a weighted adjacency matrix. Moreover, since $d_{i j}(\mathbf{x}(t))=d_{j i}(\mathbf{x}(t))$, we have that $a_{i j}(\mathbf{x}(t))=a_{j i}(\mathbf{x}(t))$, and so $A(\mathbf{x}(t))$ is symmetric.

Another way to represent the structure of a graph $\mathcal{G}(\mathbf{x}(t))$ is by a weighted Laplacian matrix

$$
L(\mathbf{x}(t))=\Delta(\mathbf{x}(t))-A(\mathbf{x}(t))
$$

where $\Delta(\mathbf{x}(t))=\operatorname{diag}\left(\sum_{j=1}^{n} a_{i j}(\mathbf{x}(t))\right)$ is the valency matrix. The spectral properties of the Laplacian matrix are closely related to graph connectivity. In particular,

Lemma 2.4 ( [19]): Let $\lambda_{1}(\mathbf{x}(t)) \leq \ldots \leq \lambda_{n}(\mathbf{x}(t))$ be the ordered eigenvalues of the Laplacian matrix $L(\mathbf{x}(t))$. Then

a) $\lambda_{1}(\mathbf{x}(t))=0$ for all $\mathbf{x}(t) \in \mathbb{R}^{n m}$, with corresponding eigenvector 1 , i.e., the vector of all entries equal to 1 ;

b) $\lambda_{2}(\mathbf{x}(t))>0$ if and only if $\mathcal{G}(\mathbf{x}(t))$ is connected.

Using Lemma 2.4(b) we can define the set $\mathcal{X}_{\mathcal{C}_{n}}$ of desired states corresponding to graphs in $\mathcal{C}_{n}$ as

$$
\mathcal{X}_{\mathcal{C}_{n}}=\left\{\mathbf{x}(t) \in \mathbb{R}^{n m} \mid \lambda_{2}(\mathbf{x}(t))>0\right\} .
$$

So Problem 1 can be reformulated as

Problem 2 (Algebraic Formulation): Let $\mathcal{X}_{\mathcal{C}_{n}}$ be the desired set of states, and assume any input to the graph $\mathcal{G}(\mathbf{x}(t))$ in the form of node motion. Given $\mathcal{X}_{\mathcal{C}_{n}}$, determine a control law $\mathbf{u}(t)$ so that if $\mathbf{x}(0) \in \mathcal{X}_{\mathcal{C}_{n}}$, then $\mathbf{x}(t) \in \mathcal{X}_{\mathcal{C}_{n}}$ for all $t \geq 0$.

\footnotetext{
${ }^{2}$ Note that any other smooth function with similar properties can be used.
} 


\section{POTENTIAL FIELD FOR GRAPH CONNECTIVITY}

Let $\mathcal{G}(\mathbf{x}(t))$ be a dynamic graph and $L(\mathbf{x}(t))$ its weighted Laplacian matrix as defined in (3). Let $P$ be a fixed $n \times(n-1)$ matrix with column structure $P=\left[\begin{array}{llll}p_{1} & p_{2} \ldots p_{n}\end{array}\right]$ such that $p_{i}^{T} p_{j}=0$ for all $i, j=$ $1, \ldots, n$ and $p_{i}^{T} \mathbf{1}=0$ for all $i=1, \ldots, n$, where $\mathbf{1}$ denotes an $n$ dimensional vector with all entries equal to $1 .^{3}$ By Lemma 2.4(a), $L(\mathbf{x}(t))$ is positive semidefinite for all $\mathbf{x}(t) \in \mathbb{R}^{n m}$, and hence, we have the following result for the expression $P^{T} L(\mathbf{x}) P{ }^{4}$

Proposition 3.1: $P^{T} L(\mathbf{x}) P \succeq 0$ for all $\mathbf{x} \in \mathbb{R}^{n m}$.

Proof: Let $\mathbf{z} \in \mathbb{R}^{n-1}$, and consider the quadratic form $\mathbf{z}^{T} P^{T} L(\mathbf{x}) P \mathbf{z}=(P \mathbf{z})^{T} L(\mathbf{x}) P \mathbf{z}$. Let $\mathbf{w}=P \mathbf{z}$. Since $P$ is full rank, $\mathbf{w}=P \mathbf{z}$ defines an injective mapping between $\mathbb{R}^{n-1}$ and $\mathbb{R}^{n}$; and since $\mathbf{w}^{T} L(\mathbf{x}) \mathbf{w} \geq 0$ for all $\mathbf{w} \in \mathbb{R}^{n}$ and all $\mathbf{x} \in \mathbb{R}^{n m}$, we conclude that $\mathbf{z}^{T} P^{T} L(\mathbf{x}) P \mathbf{z} \geq 0$ for all $\mathbf{z} \in \mathbb{R}^{n-1}$, and all $\mathbf{x} \in \mathbb{R}^{n m}$ which completes the proof.

The following proposition characterizes the eigenvalues of $P^{T} L(\mathbf{x}) P$.

Proposition 3.2 : Let $0=\lambda_{1}(\mathbf{x}) \leq \lambda_{2}(\mathbf{x}) \leq \ldots \leq \lambda_{n}(\mathbf{x})$ be the ordered eigenvalues of the Laplacian matrix $L(\mathbf{x})$. Then, $\lambda_{2}(\mathbf{x}) \leq$ $\ldots \leq \lambda_{n}(\mathbf{x})$ are the eigenvalues $P^{T} L(\mathbf{x}) P$.

Proof: The proof of this result is based on the relation between the characteristic polynomials of $L(\mathbf{x})$ and $P^{T} L(\mathbf{x}) P$. Since

$$
\left[\begin{array}{c}
P^{T} \\
\frac{1}{\|\mathbf{1}\|} \mathbf{1}^{T}
\end{array}\right]\left[P \frac{1}{\|\mathbf{1}\|} \mathbf{1}\right]=I_{n}
$$

we have that,

$$
\begin{aligned}
& \operatorname{det}(L(\mathbf{x})-s I)=\operatorname{det}\left[\begin{array}{c}
P^{T} \\
\frac{1}{\|\mathbf{1}\|} \mathbf{1}^{T}
\end{array}\right](L(\mathbf{x})-s I)\left[P \frac{1}{\|\mathbf{1}\|} \mathbf{1}\right] \\
& =\operatorname{det}\left[\begin{array}{cc}
P^{T}(L(\mathbf{x})-s I) P & 0 \\
0 & -s
\end{array}\right] \\
& =-s \operatorname{det} P^{T}(L(\mathbf{x})-s I) P
\end{aligned}
$$

which completes the proof.

A sufficient condition that guarantees that $\mathbf{x}(t) \in \mathcal{X}_{\mathcal{C}_{n}}$ for all $t \geq 0$ is to choose $\mathbf{u}(t)$ so that $\lambda_{2}(\mathbf{x}(t))$ is maximized over time. This approach is followed in [4] where the authors discretize the problem, and at every time step, solve the semidefinite program $\max \left\{\epsilon \mid P^{T} L(\mathbf{x}(t)) P \succeq \epsilon I_{n}\right\}$. In this paper, we choose a different approach, and construct a potential field that "blows up" when the state $\mathbf{x}(t)$ approaches the boundary of the free space $\mathcal{X}_{\mathcal{C}_{n}}$, defined as $\left.\partial \mathcal{X}_{\mathcal{C}_{n}}=\left\{\mathbf{x}(t) \in \mathbb{R}^{n m}\right) \mid \lambda_{2}(\mathbf{x}(t))=0\right\}$. Since $\lambda_{2}(\mathbf{x}(t))$ is not a differentiable function, ${ }^{5}$ we use the fact that $\lambda_{2}(\mathbf{x}(t))$ is positive if and only if the determinant of the matrix $P^{T} L(\mathbf{x}(t)) P$ is positive, which enables us to equivalently represent the set of desired states $\mathcal{X}_{\mathcal{C}_{n}}$ in (4) as

$$
\mathcal{X}_{\mathcal{C}_{n}}=\left\{\mathbf{x}(t) \in \mathbf{R}^{n m} \mid \operatorname{det}\left(P^{T} L(\mathbf{x}(t)) P\right)>0\right\} .
$$

Hence, we can define a potential field on the set $\mathcal{X}_{\mathcal{C}_{n}}$, and use its negative gradient as the control input to navigate the multiagent system

\footnotetext{
${ }^{3}$ A possible choice for $P$ could be the matrix $I_{n}-\frac{1}{n} \mathbf{1 1}^{T}$ where $I_{n}$ is the $n$-dimensional identity matrix. Observe that $\operatorname{rank}\left(I_{n}-\frac{1}{n} \mathbf{1 1}^{T}\right)=n-1$ and $\left(I_{n}-\frac{1}{n} \mathbf{1 1}^{T}\right) \mathbf{1}=\mathbf{0}$. Hence, the columns of $I_{n}-\frac{1}{n} \mathbf{1 1}^{T}$ span the space $\mathbf{1}^{\perp}$ and we can use Gram-Schmidt orthonormalization to get $P$.

${ }^{4}$ To simplify notation, we drop the dependence of the state $\mathbf{x}$ on time $t$.

${ }^{5}$ By Propositions 3.1 and $3.2, \lambda_{2}(\mathbf{x})$ can be written as
}

$$
\lambda_{2}(\mathbf{x})=\inf _{\mathbf{z} \in \mathbf{1}^{\perp}} \frac{\mathbf{z}^{T} L(\mathbf{x}) \mathbf{z}}{\mathbf{z}^{T} \mathbf{z}}=\inf _{\mathbf{z} \neq \mathbf{0}} \frac{\mathbf{z}^{T} P^{T} L(\mathbf{x}) P \mathbf{z}}{\mathbf{z}^{T} \mathbf{z}} .
$$

away from the undesired set $\partial \mathcal{X}_{\mathcal{C}_{n}}$. In particular, we have the following proposition.

Proposition 3.3: Define the potential field

$$
\phi_{c}(\mathbf{x}(t))=\frac{1}{\left(\operatorname{det}\left(P^{T} L(\mathbf{x}(t)) P\right)\right)^{a}}
$$

where $a$ is any positive constant. Then, the control $\mathbf{u}_{c}(t)=$ $-\nabla_{\mathbf{x}} \phi_{c}(\mathbf{x}(t))$ guarantees that $\mathbf{x}(t) \in \mathcal{X}_{\mathcal{C}_{n}}$ for all $t \geq 0$.

Proof: By Proposition 3.2, we have that $\operatorname{det}\left(P^{T} L(\mathbf{x}) P\right)=$ $\prod_{i=2}^{n} \lambda_{i}(\mathbf{x})$, and hence, $\lambda_{2}(\mathbf{x})>0$ if and only if $\operatorname{det}\left(P^{T} L(\mathbf{x}) P\right)>0$ by positive semidefiniteness of $P^{T} L(\mathbf{x}) P$. Clearly, $\phi_{c}\left(\partial \mathcal{X}_{\mathcal{C}_{n}}\right)=\infty$ and $\phi_{c}\left(\mathcal{X}_{\mathcal{C}_{n}}\right)<\infty$. Hence, $\phi_{c}\left(\mathcal{X}_{\mathcal{C}_{n}}\right)<\phi_{c}\left(\partial \mathcal{X}_{\mathcal{C}_{n}}\right)$, and since for all $\mathbf{x} \in \mathcal{X}_{\mathcal{C}_{n}}$ we have that $\dot{\phi}_{c}(\mathbf{x})=-\left\|\nabla_{\mathbf{x}} \phi_{c}(\mathbf{x})\right\|^{2} \leq 0$, we conclude that the set $\mathcal{X}_{\mathcal{C}_{n}}$ is an invariant of motion for the multiagent system, i.e., the graph will always remain connected.

In other words, we treat graph connectivity as anstacle in the free space, and move in the direction of the negative gradient of the potential field. Note, that the controller in Proposition 3.3 guarantees that $\lambda_{2}(\mathbf{x})>0$ for all time but not that $\lambda_{2}(\mathbf{x})$ will also be maximized as is the case in [4], since even if $\operatorname{det}\left(P^{T} L(\mathbf{x}) P\right)=\prod_{i=2}^{n} \lambda_{i}(\mathbf{x})$ increases, $\lambda_{2}(\mathbf{x})$ can decrease. On the other hand, observe that $\lambda_{2}(\mathbf{x})$ will attain its global maximum if and only if $\operatorname{det}\left(P^{T} L(\mathbf{x}) P\right)$ will also attain its global maximum. ${ }^{6}$ However, since $L(\mathbf{x})$ is a function of the state vector $\mathbf{x}$ through the sigmoid function [see (2)-(3)], $\operatorname{det}\left(P^{T} L(\mathbf{x}) P\right)$ is not a concave function of the state $\mathbf{x}$, and so only convergence to a local maximum is guaranteed. We conclude this section by providing a closed form expression for the controller in Proposition 3.3.

Proposition 3.4: The control law

$$
\mathbf{u}_{c}(t)=\frac{a}{(\operatorname{det} M(\mathbf{x}(t)))^{a}}\left[\begin{array}{c}
\operatorname{tr}\left[M^{-1}(\mathbf{x}(t)) \frac{d}{d x_{1}} M(\mathbf{x}(t))\right] \\
\vdots \\
\operatorname{tr}\left[M^{-1}(\mathbf{x}(t)) \frac{d}{d x_{n}} M(\mathbf{x}(t))\right]
\end{array}\right]
$$

where $M(\mathbf{x})=P^{T} L(\mathbf{x}) P$, guarantees that the multiagent closed loop system $\dot{\mathbf{x}}(t)=\mathbf{u}_{c}(t)$ always remains in the set $\mathcal{X}_{\mathcal{C}_{n}}$ corresponding to connected graphs in $\mathcal{C}_{n}$.

Proof: Let $M(\mathbf{x})=P^{T} L(\mathbf{x}) P$, and denote by $c_{i j}(\mathbf{x})$ the cofactor of the entry $m_{i j}(\mathbf{x})$ of the matrix $M(\mathbf{x})$. Let $C(\mathbf{x})$ denote the cofactor matrix and $c_{i j}^{T}(\mathbf{x})$ denote the $i, j$ th entry of $C^{T}(\mathbf{x})$, i.e., $c_{i j}^{T}(\mathbf{x})=$ $c_{j i}(\mathbf{x})$. Since the determinant is a differentiable function of matrix entries, in particular, it is a sum of products of entries, the chain rule gives

$$
\frac{d}{d x_{k}} \operatorname{det} M(\mathbf{x})=\sum_{i=1}^{n-1} \sum_{j=1}^{n-1}\left(\frac{\partial}{\partial m_{i j}} \operatorname{det} M(\mathbf{x})\right) \frac{d}{d x_{k}} m_{i j}(\mathbf{x}) .
$$

For all $j=1, \ldots, n-1$, computation of the Laplace expansion of the determinant along the $j$ th column gives det $M(\mathbf{x})=$ $\sum_{i=1}^{n-1} c_{i j}(\mathbf{x}) m_{i j}(\mathbf{x})$, and hence, $\frac{\partial}{\partial m_{i j}} \operatorname{det} M(\mathbf{x})=c_{i j}(\mathbf{x})$. Therefore,

$$
\begin{aligned}
\frac{d}{d x_{k}} \operatorname{det} M(\mathbf{x}) & =\sum_{i=1}^{n-1} \sum_{j=1}^{n-1} c_{i j}(\mathbf{x}) \frac{d}{d x_{k}} m_{i j}(\mathbf{x}) \\
& =\sum_{j=1}^{n-1} \sum_{i=1}^{n-1} c_{j i}^{T}(\mathbf{x}) \frac{d}{d x_{k}} m_{i j}(\mathbf{x})=\operatorname{tr}\left[C^{T}(\mathbf{x}) \frac{d}{d x_{k}} M(\mathbf{x})\right] .
\end{aligned}
$$

A direct consequence of the Laplce expansion of the determinant is the identify $I$ det $M(\mathbf{x})=M(\mathbf{x}) C^{T}(\mathbf{x})$. Proposition 3.3 guarantees that

\footnotetext{
${ }^{6}$ Note that the eigenvalues of the Laplacian matrix are upper bounded, and hence, so is the determinant.
} 
$\lambda_{2}(\mathbf{x}(t))>0$ for all time, and so det $M(\mathbf{x})>0$ for all $\mathbf{x} \in \mathbb{R}^{n m}$. Thus, $M(\mathbf{x})$ is always positive definite, and hence, invertible. Therefore, by left multiplication of the previous identify by $M^{-1}(\mathbf{x})$, we get $M^{-1}(\mathbf{x}) \operatorname{det} M(\mathbf{x})=C^{T}(\mathbf{x})$, and substituting in the expression for $\frac{d}{d x_{k}} \operatorname{det} M(\mathbf{x})$ we get

$$
\begin{aligned}
\frac{d}{d x_{k}} \operatorname{det} M(\mathbf{x}) & =\operatorname{tr}\left[(\operatorname{det} M(\mathbf{x})) M^{-1}(\mathbf{x}) \frac{d}{d x_{k}} M(\mathbf{x})\right] \\
& =\operatorname{det} M(\mathbf{x}) \operatorname{tr}\left[M^{-1}(\mathbf{x}) \frac{d}{d x_{k}} M(\mathbf{x})\right]
\end{aligned}
$$

where $M^{-1}(\mathbf{x})=\left(P^{T} L(\mathbf{x}) P\right)^{-1}$ and $\frac{d}{d x_{k}} M(\mathbf{x})=P^{T} \frac{d}{d x_{k}} L(\mathbf{x}) P$, and the result follows directly from Proposition 3.3 and a simple application of the chain rule.

\section{CONNECTIVITY TASKS}

In Section III, we developed a controller based on artificial potential fields that by construction, guarantees that the network of agents remains connected for all time. Due to its feedback nature, our controller is more robust and more flexible to account for secondary objectives, in contrast to optimization-based approaches [1], [4]. In particular, depending on whether the objective is "repulsive" (collision avoidance) or "attractive" (convergence to a point), it can be, in general, included in the denominator or the numerator of the modified potential function, respectively. However, any modified controller should be studied to see whether it has guaranteed properties. For particular cases, such as stationary obstacle avoidance, this could be a challenging process. In this section, we first show that our controller maintains (and in particular increases) connectivity of the multiagent system, and then, consider a nontrivial connectivity task involving collision avoidance and single leader tracking objectives.

\section{A. Maintaining Connectivity}

In this connectivity task we consider a group of $n=20$ agents (dots), that are initially, randomly distributed in the plane, so that the initial underlying graph is connected. Whenever two agents are adjacent in the corresponding graph, an edge is drawn between them. Under the control law in (6), the connectivity of the multiagent system is maintained, and in particular, is increased leading to a closed loop solution to the rendezvous problem [15], [16]. However, as discussed in Section III, the gradient descent controller (6) does not provide guarantees for rendezvous. These results are illustrated in Fig 2.

\section{B. Tracking a Leader with Collision Avoidance Constraints}

1) Collision Avoidance: Many potential field approaches have been proposed in the literature that guarantee collision avoidance for multiagent systems [10], [11]. In this paper, we choose to adopt the one introduced in [10] due to its "local" nature. This is because, unlike connectivity maintenance, which imposes maximum distance constraints on the agents, collision avoidance imposes minimum distance constraints on the agents. Hence, we are dealing with two conflicting objectives; and in order to resolve this problem, we need to make the collision avoidance objective ineffective once the agents are sufficiently far from one another. In particular, for every pair $(i, j)$ of agents, the authors in [10], define the function (Fig. 3)

$$
\beta_{i j}(\mathbf{x}(t))=\left(1-\mu \frac{\left(\left\|x_{i}(t)-x_{j}(t)\right\|^{2}-d^{2}\right)^{2}}{1+\left(\left\|x_{i}(t)-x_{j}(t)\right\|^{2}-d^{2}\right)^{2}}\right)^{\rho}
$$

where $\mu=\left(1+d^{4}\right) / d^{4}$ and $\rho=\left[1-\operatorname{sign}\left(\left\|x_{i}(t)-x_{j}(t)\right\|-d\right)\right] / 2$. This function varies in the interval $[0,1]$, becoming 0 when the distance $\left\|x_{i}(t)-x_{j}(t)\right\|$ is 0 , and attaining its maximum value 1 when
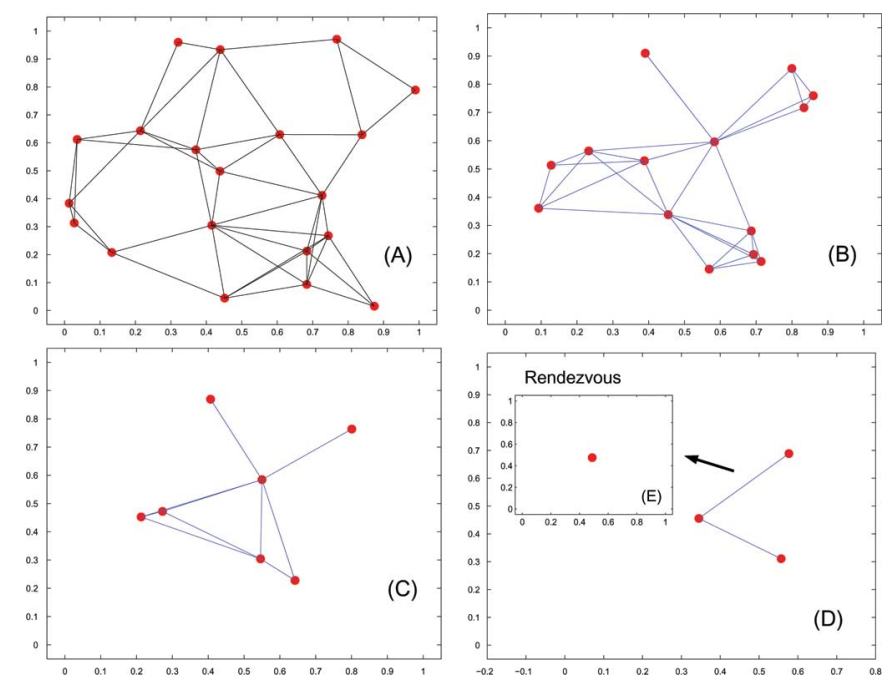

Fig. 2. Rendezvous for 20 agents.

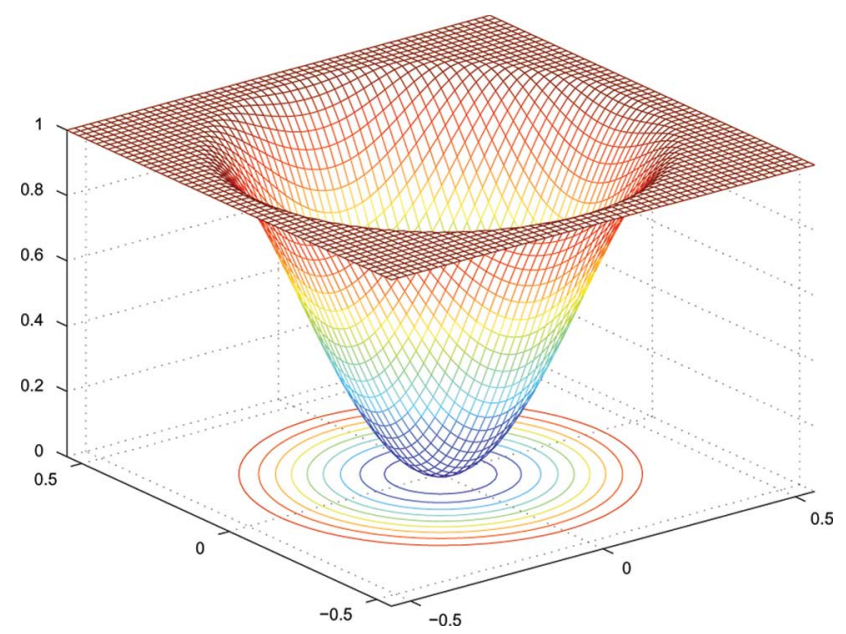

Fig. 3. Plot of the function $\beta_{i j}\left(\left\|x_{i}(t)-x_{j}(t)\right\|\right)$ for $d=0.5$.

$\left\|x_{i}(t)-x_{j}(t)\right\|$ is larger than $d$. In this way, the presence of other agents on the motion of an agent remains "local," within a region of radius $d$. Then, the net effect of all neighboring distances in the multiagent system is captured in the function $\beta(\mathbf{x}(t))=\prod_{i, j} \beta_{i j}(\mathbf{x}(t))$. Hence, we can define the potential field for collision avoidance $\phi_{a}(\mathbf{x}(t))=1 /\left[\beta(\mathbf{x}(t))^{k}\right]$, with $k>0$ a constant, which composed with the connectivity potential field of Proposition 3.3 gives rise to the control law

$$
\mathbf{u}(t)=-\nabla_{\mathbf{x}} \phi(\mathbf{x}(t))=-\nabla_{\mathbf{x}}\left(\phi_{c}(\mathbf{x}(t))+\phi_{a}(\mathbf{x}(t))\right)
$$

Clearly, $\phi(\mathbf{x}(t)) \rightarrow \infty$ whenever $\lambda_{2}(\mathbf{x}(t)) \rightarrow 0$ or $\beta(\mathbf{x}(t)) \rightarrow 0$, and thus, moving in the direction of the negative gradient of $\phi(\mathbf{x}(t))$, guarantees that the graph will remain connected and collisions between the agents will be avoided.

2) Tracking a Leader: Consider now the case where agent $i$ is a leader for a group of $n$ agents, with dynamics independent of the other $n-1$ agents. We show, that under the control law (7), the agents track the leader and maintain a connected formation while avoiding collisions with each other. The following simulation demonstrates the aforementioned connectivity task. We consider a network of $n=9$ agents with a leader labeled "L," having dynamics of the form

$$
u_{L}=\left[\begin{array}{l}
1+x_{L, 2}\left(1-\frac{3}{2} x_{L, 2}\right)-x_{L, 1}^{3} \\
1-x_{L, 1}\left(1+x_{L, 1}^{2}\right)-\frac{3}{2} x_{L, 2}^{2}
\end{array}\right]
$$



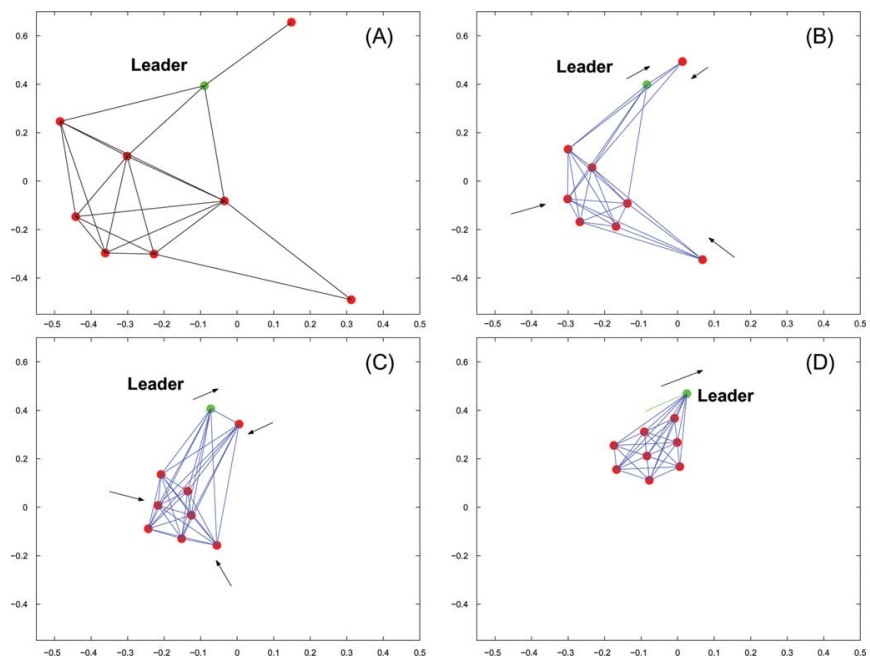

Fig. 4. One Leader and eight Followers (initial phase).

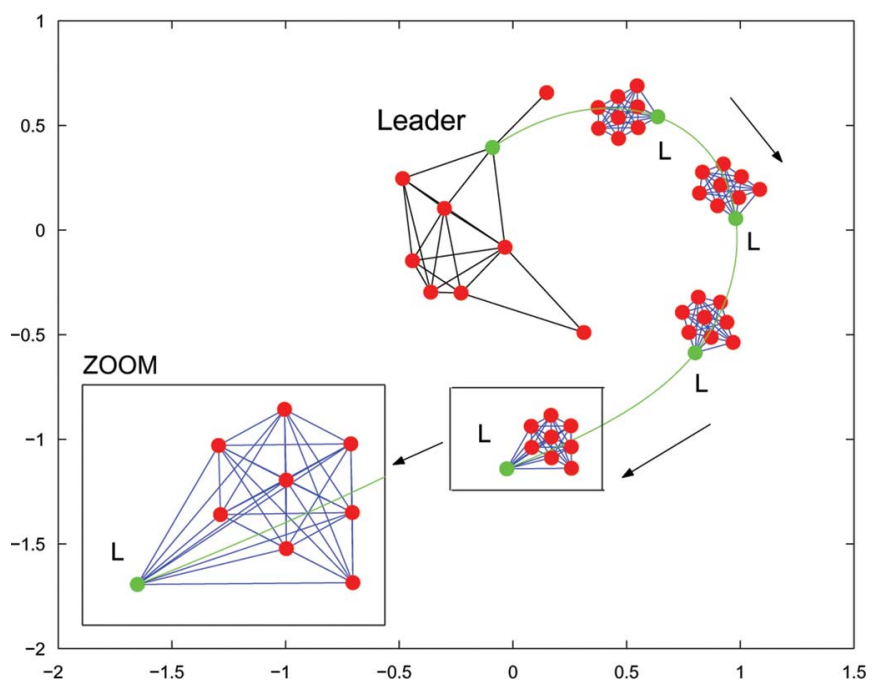

Fig. 5. One Leader (labeled "L") and eight Followers (tracking leader phase).

The remaining eight agents have controllers defined as in (7). Initially, all agents (dots) are randomly distributed in the plane so that the initial network is connected, and an edge is drawn between any two connected agents. Figs. 4 and 5 illustrate our results for connectivity parameters $\epsilon=0.25$ and $w=10$ and collision avoidance radius $d=0.1$. Observe that the imposed system specifications are always satisfied.

\section{CONCLUSION}

In this paper, we considered the problem of controlling the structure of dynamic graphs so that the resulting motion always preserves connectivity and collision avoidance properties. The idea was to define artificial potential fields where both objectives were modeled as obstacles in the free space and move in the direction of their negative gradient. Connectivity was captured by the smallest eigenvalue of the projected graph Laplacian matrix to the space perpendicular to the Laplacian eigenvector of ones. Compared to our previous work [1], this approach is more robust due to its feedback nature and more amenable in accounting for secondary objectives.

\section{ACKNOWLEDGMENT}

The authors would like to thank A. Jadbabaie (University of Pennsylvania) and M. C. De Gennaro (Università del Sannio, Italy) for their discussions on dynamic graphs' connectivity.

\section{REFERENCES}

[1] M. M. Zavlanos and G. J. Pappas, "Controlling connectivity of dynamic graphs," in Proc. 44th IEEE Conf. Decision Control, Seville, Spain, Dec. 2005, pp. 6388-6393.

[2] D. P. Spanos and R. M. Murray, "Robust connectivity of networked vehicles," in Proc. 43rd IEEE Conf. Decision Control, Bahamas, Dec. 2004, pp. 2893-2898.

[3] M. Mesbahi, "On state-dependent dynamic graphs and their controllability properties," IEEE Trans. Autom. Control, vol. 50, no. 3, pp. 387-392, Mar. 2005.

[4] Y. Kim and M. Mesbahi, "On maximizing the second smallest eigenvalue of a state-dependent graph Laplacian," IEEE Trans. Autom. Control, vol. 51, no. 1, pp. 116-120, Jan. 2006.

[5] A. Jadbabaie, J. Lin, and A. S. Morse, "Coordination of groups of mobile autonomous agents using nearest neighbor rules," IEEE Trans. Autom. Control, vol. 48, no. 6, pp. 988-1001, Jun. 2003.

[6] R. Olfati-Saber and R. M. Murray, "Consensus problems in networks of agents with switching topology and time-delays," IEEE Trans. Autom. Control, vol. 49, no. 9, pp. 1520-1533, Sep. 2004.

[7] H. G. Tanner, A. Jadbabaie, and G. J. Pappas, "Flocking in fixed and switching networks," IEEE Trans. Autom. Control, vol. 52, no. 5, pp. 863868, May 2007.

[8] J. P. Desai, J. P. Ostrowski, and V. Kumar, "Modeling and control of formations of nonholonomic mobile robots," IEEE Trans. Robot. Autom., vol. 17, no. 6, pp. 905-908, Dec. 2001.

[9] P. Ögren, M. Egerstadt, and X. Hu, "A control Lyapunov function approach to multi-agent coordination," IEEE Trans. Robot. Autom., vol. 18, no. 5, pp. 847-851, Oct. 2002.

[10] A. Kumar and H. G. Tanner, "Decentralized formation stabilization and collision avoidance using navigation functions," IEEE Trans. Robot., to be published.

[11] D. V. Dimarogonas, S. G. Loizou, K. J. Kyriakopoulos, and M. M. Zavlanos, "A feedback stabilization and collision avoidance scheme for multiple independent non-point agents," Automatica, vol. 42, no. 2, pp. 229243, Feb. 2006.

[12] R. Sepulchre, D. Paley, and N. E. Leonard, "Stabilization of planar collective motion: All-to-all communication," IEEE Trans. Autom. Control, vol. 52, no. 5, pp. 811-824, May 2007.

[13] G. Lafferriere, A. Williams, J. Caughman, and J. J. P. Veerman, "Decentralized control of vehicle formations," Syst. Control Lett., vol. 54, no. 9, pp. 899-910, Sep. 2005.

[14] T. Balch and R. C. Arkin, "Behavior-based formation control for multirobot teams," IEEE Trans. Robot. Autom., vol. 14, no. 6, pp. 926-939, Dec. 1998.

[15] J. Lin, A. S. Morse, and B. D. O. Anderson, "The multi-agent rendezvous problem," in Proc. 42nd IEEE Conf. Decision Control, Maui, HI, Dec. 2003, pp. 1508-1513.

[16] J. Cortes, S. Martinez, and F. Bullo, "Robust rendezvous for mobile autonomous agents via proximity graphs in arbitrary dimensions," IEEE Trans. Autom. Control, vol. 51, no. 8, pp. 1289-1298, Aug. 2006.

[17] W. Ren and R. Beard, "Consensus of information under dynamically changing interaction topologies," in Proc. Am. Control Conf., Boston, MA, Jun. 2004, pp. 4939-4944.

[18] S. Poduri and G. S. Sukhatme, "Constrained coverage for mobile sensor networks," in Proc. IEEE Int. Conf. Robot. Autom., New Orleans, LA, May 2004, pp. 165-172.

[19] N. Biggs, Algebraic Graph Theory. Cambridge, U.K.: Cambridge Univ. Press, 1993. 